\title{
Effects of astaxanthin supplementation and electrical stimulation on muscle atrophy and decreased oxidative capacity in soleus muscle during hindlimb unloading in rats
}

\author{
Miho Kanazashi ${ }^{1} \cdot$ Masayuki Tanaka $^{2} \cdot$ Ryosuke Nakanishi $^{3} \cdot$ Noriaki Maeshige $^{4} \cdot$ Hidemi Fujino $^{4}$
}

Received: 16 January 2019 / Accepted: 24 June 2019 / Published online: 4 July 2019

(c) The Physiological Society of Japan and Springer Japan KK, part of Springer Nature 2019

\begin{abstract}
The effects of a combination of the antioxidant astaxanthin (AX) and electrical stimulation (ES) on muscle mass and mitochondrial oxidative capacity were investigated in the soleus muscle of hindlimb unloaded rats. Five groups of male SpragueDawley rats were used; control, 1-week hindlimb unloading (HU), HU + AX, HU + ES, and HU + AX + ES. Respective rats in the AX groups received 50-mg/kg AX twice daily during HU. Calf muscles of rats in the ES groups were electrically stimulated for 240 s/day during HU. One-week HU decreased muscle mass along with decreased FoxO3a phosphorylation and increased ubiquitinated proteins expressions, decreased oxidative enzymatic activity accompanied with decline in PGC-1 $\alpha$ protein expression, and increased reactive oxygen species production. However, the combination treatment could synergistically attenuate/suppress all HU-related changes, suggesting protective effects on muscle atrophy and decreased muscle oxidative capacity due to chronic neuromuscular inactivity.
\end{abstract}

Keywords Skeletal muscle $\cdot$ Atrophy $\cdot$ Oxidative stress $\cdot$ Electrical stimulation $\cdot$ Astaxanthin

\section{Introduction}

Chronic neuromuscular inactivity induces quantitative and qualitative changes in skeletal muscles. Muscle disuse results in morphological and functional alterations such as muscle atrophy [1], slow-to-fast fiber switching [2, 3], capillary regression [4-6], and mitochondrial dysfunction [7] within the muscles, particularly in muscles predominantly comprising slow oxidative fibers such as the soleus muscle.

Hidemi Fujino

fujino@phoenix.kobe-u.ac.jp

1 Department of Physical Therapy, Faculty of Health and Welfare, Prefectural University of Hiroshima, 1-1 Gakuen-cho, Mihara-shi, Hiroshima 723-0053, Japan

2 Department of Physical Therapy, Faculty of Human Sciences, Osaka University of Human Sciences, 1-4-1 Shojaku, Settsu-shi, Osaka 566-8501, Japan

3 Department of Physical Therapy, Faculty of Rehabilitation, Kobe International University, 9-1-6 Koyocho-naka, Higashinada-ku, Kobe-shi, Hyogo 658-0032, Japan

4 Department of Rehabilitation Science, Kobe University Graduate School of Health Sciences, 7-10-2 Tomogaoka, Suma-ku, Kobe-shi, Hyogo 654-0142, Japan
All of these changes in the unloaded muscles impair the contractile force [8] and oxidative capacity [2], resulting in muscle weakness and exercise intolerance. Therefore, therapeutic strategies targeting both quantitative and qualitative alterations are required to treat disuse-induced muscle fragility.

Oxidative stress results from the muscle disuse including hindlimb unloading (HU) $[9,10]$. Reportedly, disuseinduced activation of oxidative stress signaling is associated with the progression of muscle fragility resulting from conditions such as proteolysis [11], cell apoptosis [12], capillary regression [6], and decreased oxidative capacity [13]. Thus, various strategies such as antioxidant supplementation have been formulated for treating oxidative stress. Emerging studies suggest that providing antioxidant supplementation during $\mathrm{HU}$ counteracts qualitative alterations such as fibertype transition and reduced mitochondrial activity [5, 14, 15]. However, the positive effect of antioxidant treatment on muscle fiber size is still controversial. Some studies [16-18] showed its efficacy in preventing muscle atrophy whereas others [14, 15, 19-21] failed to demonstrate similar results. These inconsistent findings necessitate the investigation of the effect of antioxidant treatment along with another 
countermeasure to effectively prevent muscle atrophy due to muscle disuse.

Electrical stimulation (ES) is a therapeutic method that induces contractile activity in skeletal muscles without voluntary exercise. In addition, it is a well-established method for treating muscle atrophy due to unloading [22, 23]. Reportedly, ES during HU can attenuate muscle mass and fiber size reduction by counteracting disuse-induced activation of proteolytic signaling [24]. Although numerous studies report the efficacy of ES for preventing muscle atrophy [22-26], little is known about its effects on oxidative capacity, such as mitochondrial enzymatic activity, in the atrophying muscle. A successful prevention of muscle atrophy requires the application of ES with high-intensity training such as resistance training [22-26]. Conversely, for restoring muscle oxidative capacity, low-intensity and longduration muscle contractile activity such as endurance training is classically recommended [27-29]. Therefore, resistance training with ES that targets muscle atrophy alone may minimally affect the muscle mitochondrial oxidative activity.

Considering these reported findings, we hypothesized that a combination therapy involving antioxidant astaxanthin (AX) and ES may be able to positively influence the oxidative capacity and prevent muscle atrophy in an unloaded muscle. Therefore, we investigated the effect of this combination treatment on muscle fiber size and oxidative capacity in the soleus muscle during $\mathrm{HU}$ in rats.

\section{Materials and methods}

\section{Animals and protocol}

Ten-week-old male Sprague-Dawley (SD) rats (Japan SLC, Shizuoka, Japan) weighing 400-444 g were used. The rats were randomly divided into five groups as follows: control (Con; $n=6), \mathrm{HU}(n=6), \mathrm{HU}+\mathrm{AX}(n=6), \mathrm{HU}+\mathrm{ES}(n=6)$, $\mathrm{HU}+\mathrm{AX}+\mathrm{ES}(n=6)$ groups. All rats were housed at $22{ }^{\circ} \mathrm{C} \pm 2{ }^{\circ} \mathrm{C}$ with a $12 \mathrm{~h}$ light/dark cycle and were provided standard rodent chow and water ad libitum. This study was approved by the Institutional Animal Care and Use Committee and was performed according to the Kobe University Animal Experimentation Regulations. All experiments were conducted in accordance with the National Institute of Health Guide for the Care and Use of Laboratory Animals (National Research Council, 1996).

\section{HU}

Rats in the $\mathrm{HU}, \mathrm{HU}+\mathrm{AX}, \mathrm{HU}+\mathrm{ES}$, and $\mathrm{HU}+\mathrm{AX}+\mathrm{ES}$ groups subjected to $\mathrm{HU}$ for a week via tail suspension as previously described $[1,30]$. Briefly, by avoiding any weight on the hindlimbs, these rats were suspended by the tail to prevent hindlimb contact with the floor or side of the cage. Rats were allowed to move freely in the cage using their forearms and access food and water ad libitum.

\section{AX supplementation}

Rats in the $\mathrm{HU}+\mathrm{AX}$ and $\mathrm{HU}+\mathrm{AX}+\mathrm{ES}$ groups were orally administered AX oil (Fuji Chemical Industry Co. Ltd, Toyama, Japan) using a feeding needle twice daily (50 mg/kg) with a 9-h interval between two doses (total of $100 \mathrm{mg} / \mathrm{kg}$ ) day) during 1 week of unloading. Rats in the Con, HU, and $\mathrm{HU}+\mathrm{ES}$ groups were similarly administered oil without AX $[15,20]$.

\section{ES protocol}

Rats in the $\mathrm{HU}+\mathrm{ES}$ and $\mathrm{HU}+\mathrm{AX}+\mathrm{ES}$ groups were subjected to ES twice daily during 1 week of unloading. These animals were anesthetized before ES with pentobarbital sodium (40 mg/kg body weight, intraperitoneally). To exclude the influences of repeated periods of anesthesia and changing posture, the rats in the Con, $\mathrm{HU}$, and $\mathrm{HU}+\mathrm{AX}$ groups were subjected to an anesthetic and postural changing protocol identical to those in the electrical stimulated groups. For transcutaneously treating the soleus muscle, an electrical stimulator (ES-360, Ito, Tokyo, Japan) was used as previously described [22]. One surface electrode (1-cm diameter) was mounted on the medial and lateral calf muscles each. ES was achieved through a sinusoidally modulated waveform, and the current was delivered at a frequency of $100 \mathrm{~Hz}$. Stimulation at this frequency reportedly results in a forceful contraction of the soleus muscle [22]. Current intensity was set daily to generate a supramaximal contraction. One burst of ES was performed every $3 \mathrm{~s}$ (time on: $1 \mathrm{~s}$; time off $2 \mathrm{~s}$ ) for $1 \mathrm{~min}$, followed by $5 \mathrm{~min}$ of rest. Six consecutive stimulation sessions were conducted twice daily, resulting in a total daily ES time of $240 \mathrm{~s}$, which is reported to be partly effective for attenuating disuse-induced soleus atrophy [22, 24].

\section{Sample extraction}

At the end of the 1-week experimental period, the animals were euthanized by administering an overdose of sodium pentobarbital. The soleus muscle was removed, weighed, and immediately frozen in an isopentane/dry ice bath and stored at $-80{ }^{\circ} \mathrm{C}$ until subsequent histological and biochemical analyses.

\section{Histochemical analysis}

Soleus muscles were sliced into $12-\mu \mathrm{m}$ thick transverse sections using a cryostat microtome (CM-1510S, Leica 
Microsystems, Mannheim, Germany). Sections were stained using succinate dehydrogenase (SDH) to assess the fiber cross-sectional area (FCSA) and skeletal muscle oxidative capacity as previously described [2, 15, 31]. Briefly, the sections were incubated in 0.1-M phosphate buffer ( $\mathrm{pH}$ 7.6) containing 0.9-mM NaN 3 , 0.9-mM 1-methoxyphenazine methylsulfate, $1.5-\mathrm{mM}$ nitroblue tetrazolium, 5.6-mM EDTA-disodium salt, and 48-mM succinate disodium salt for $45 \mathrm{~min}$ at $37^{\circ} \mathrm{C}$. Densitometric analysis was performed to determine SDH activity in each group. Mean FCSA and SDH activity were determined for 400-500 fibers per muscle using microscopic images of SDH staining. The other sections were stained with alkaline phosphatase (AP) as previously described to observe the capillarity of the soleus muscles [15]. Briefly, the sections were incubated in 5-bromo-4-chloro-3-indolyl phosphate/nitro blue tetrazolium for $45 \mathrm{~min}$ at $37{ }^{\circ} \mathrm{C}$ and fixed with $4 \%$ paraformaldehyde. The capillary-to-fiber $(\mathrm{C} / \mathrm{F})$ ratio was determined by counting capillaries and myofibers on each cryosection using the microscopic images. For the measurement of $\mathrm{C} / \mathrm{F}$ ratio, more than 240 muscle fibers were measured per muscle. All measurements were performed using the NIH ImageJ software program (NIH, Bethesda, MD, USA).

\section{Dihydroethidium staining}

Oxidative fluorescent dihydroethidium (DHE) probe was used for measuring reactive oxygen species (ROS) production within the soleus muscle as previously described [15, 20, 32-34]. Briefly, soleus muscles were sliced into $10-\mu \mathrm{m}$ thick transverse sections using a cryostat microtome. Subsequently, the sections were air-dried, incubated with 5- $\mu \mathrm{M}$ DHE probe (Wako Pure Chemicals, Osaka, Japan) for 30 min at $37{ }^{\circ} \mathrm{C}$ in a dark box, rinsed with $37{ }^{\circ} \mathrm{C}$ PBS and then mounted with VECTASHIELD mounting medium with DAPI (H-1200, Vector Laboratories, Burlingame, CA). DHE emits red fluorescence when oxidized to ethidium bromide, which intercalates within the nuclear DNA. Stained slides were quickly imaged with fluorescence microscope (BZX710, Keyence, Osaka, Japan) keeping the same exposure for every section with appropriate filters for DHE (excitation 490; emission $590 \mathrm{~nm}$ ) and DAPI [15, 20, 32-34]. Densitometric analysis for DHE and DAPI staining was performed with the BZ-X Analyzer (BZ-H3C, Keyence, Japan) using 4-6 images per muscle. DHE fluorescence intensity was normalized as fold of the corresponding DAPI fluorescence intensity [35].

\section{Western blotting}

A portion (approximately $20 \mathrm{mg}$ ) of each soleus muscle was homogenized in RIPA lysis buffer containing $1 \%$ (v/v) phosphatase inhibitor cocktail and $1 \%(\mathrm{v} / \mathrm{v})$ protease inhibitor cocktail to determinate the SOD-1, PGC- $1 \alpha$, and FoxO3a protein expressions. Additionally, another portion (approximately $10 \mathrm{mg}$ ) of each soleus muscle was homogenized in 5-M urea, 2-M thiourea, 10-mM sodium diphosphate decahydrate, and $0.1 \%(\mathrm{v} / \mathrm{v})$ 2-mercaptoethanol containing $1 \%$ protease inhibitor cocktail to determine ubiquitinated proteins expression. Total protein concentration in supernatants was determined using the Bradford analysis using the protein assay kit (Bio-Rad Laboratories, Hercules, CA, USA). Briefly, proteins were loaded and separated using $12.5 \%$ (for SOD-1, $20 \mu \mathrm{g} / \mathrm{lane}$ ), $10 \%$ (for ubiquitinated proteins, $40 \mu \mathrm{g} / \mathrm{lane}$ ), and $7.5 \%$ (for PGC- $1 \alpha$ and FoxO3a, $40 \mu \mathrm{g}$ / lane) SDS-polyacrylamide gels. The proteins were blotted on polyvinylidene difluoride membranes and blocked for $1 \mathrm{~h}$ with Tris-buffered saline containing Tween 20 (TBST) in 3\% bovine serum albumin. The membranes were incubated with primary antibodies against goat anti-SOD-1 (diluted 1:200 in TBST; Santa Cruz Biotechnology), mouse anti-polyubiquitin protein conjugates (diluted 1:100 in TBST; Enzo Life Sciences), rabbit anti-PGC-1 $\alpha$ (1:200 in TBST; Santa Cruz Biotechnology), rabbit anti-phosphorylated Ser253-FoxO3a (diluted 1:1000 in TBST; Cell Signaling Technology), and rabbit anti-FoxO3a (diluted 1:1000 in TBST; Cell Signaling Technology) overnight at $4{ }^{\circ} \mathrm{C}$ and then incubated in a solution with either anti-rabbit, -mouse, or -goat IgG conjugated to horseradish peroxidase (1:1000 in TBST) for $1 \mathrm{~h}$. The proteins were detected using EzWestLumi One (ATTO, Tokyo, Japan). Finally, images were captured using the LAS-1000 imaging system (Fujifilm, Tokyo, Japan) with a chemiluminescent image analyzer, and the proteins were quantified using the Multi-Gauge Image Analysis Software program (Fujifilm) against the relative concentration of $\beta$-actin (diluted 1:1000; Santa Cruz Biotechnology) as an internal control.

\section{Statistical analysis}

All values were expressed as mean and standard error of mean. Significant differences between groups were determined using one-way ANOVA followed by the Tukey's post hoc test to determine specific differences between groups. Values of $p<0.05$ were considered to be statistically significant.

\section{Results}

\section{Body weight and soleus muscle weight}

Body weights in all $\mathrm{HU}$ groups were significantly lower than that in the Con group (Table 1). The absolute soleus muscle mass in all HU groups was significantly lower than that in the Con group. Additionally, the absolute soleus mass in 
Table 1 Body weight and soleus muscle mass

\begin{tabular}{lllll}
\hline & $\begin{array}{l}\text { Initial body } \\
\text { weight }(\mathrm{g})\end{array}$ & $\begin{array}{l}\text { Final body } \\
\text { weight }(\mathrm{g})\end{array}$ & $\begin{array}{l}\text { Absolute soleus mus- } \\
\text { cle mass }(\mathrm{mg})\end{array}$ & $\begin{array}{l}\text { Relative soleus muscle } \\
\text { mass (mg/100 g BW) }\end{array}$ \\
\hline Con & $427 \pm 7$ & $459 \pm 7$ & $193 \pm 8$ & $42.1 \pm 1.5$ \\
HU & $414 \pm 3$ & $404 \pm 3^{*}$ & $120 \pm 4^{*}$ & $29.6 \pm 1.2^{*}$ \\
HU + AX & $420 \pm 4$ & $404 \pm 5^{*}$ & $132 \pm 3^{*}$ & $32.7 \pm 0.5^{*}$ \\
HU + ES & $424 \pm 2$ & $382 \pm 7^{*}$ & $139 \pm 4^{*}$ & $36.4 \pm 1.1^{\dagger}$ \\
HU + AX +ES & $419 \pm 4$ & $385 \pm 4^{*}$ & $140 \pm 3^{\dagger}$ & $36.4 \pm 0.7^{\dagger}$ \\
\hline
\end{tabular}

Values indicate mean \pm SEM

Con control group, $H U$ hindlimb unloading group, $H U+A X$ hindlimb unloading plus astaxanthin treatment group, $H U+E S$ hindlimb unloading plus electrical stimulation group, $H U+A X+E S$ hindlimb unloading plus astaxanthin treatment with electrical stimulation group, SEM standard error of mean

The symbols $*$ and ${ }^{\dagger}$ significant differences from the Con and HU groups, respectively, at $p<0.05$

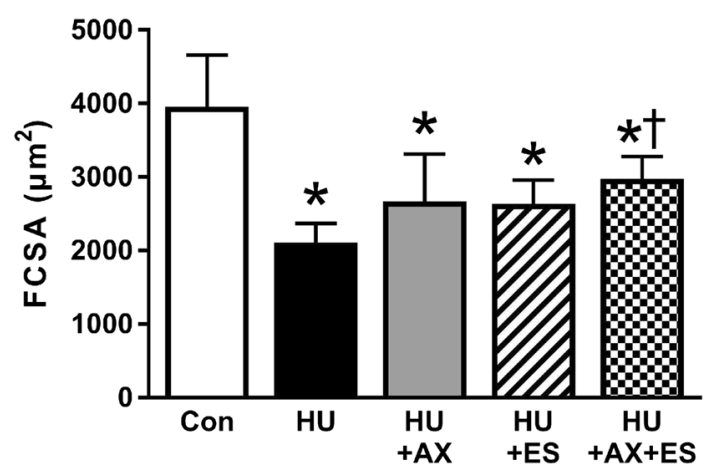

Fig. 1 Fiber cross-sectional area in each group measured from the images of SDH staining. Values indicate mean \pm SEM. The symbols $*$ and ${ }^{\dagger}$ significant differences from the Con and HU groups, respectively, at $p<0.05$. Con control group, $H U$ hindlimb unloading group, $H U+A X$ hindlimb unloading plus astaxanthin treatment group, $H U+E S$ hindlimb unloading plus electrical stimulation group, $H U+A X+E S$ hindlimb unloading plus astaxanthin treatment with electrical stimulation group, SEM standard error of mean the $\mathrm{HU}+\mathrm{AX}+\mathrm{ES}$ group was significantly higher than that in the HU group. The relative soleus muscle mass in all HU groups was significantly lower than that in the Con group. Furthermore, the relative soleus mass in the HU + ES and $\mathrm{HU}+\mathrm{AX}+\mathrm{ES}$ groups was significantly higher than that in the HU group (Table 1).

\section{FCSA}

FCSAs were measured using the microscopic images of specimens following SDH staining in each group. FCSA in all HU groups was significantly lower than that in the Con group. In addition, FCSA in the HU + AX+ES group was significantly higher than that in the HU group (Fig. 1).

\section{Ratio of phosphorylated to total Fox03a protein expression}

The ratio of phosphorylated to total FoxO3a protein expression in the HU group was significantly lower than that in the Con group. This ratio in $\mathrm{HU}+\mathrm{ES}$ and $\mathrm{HU}+\mathrm{AX}+\mathrm{ES}$ groups was significantly higher than that in $\mathrm{HU}$ and $\mathrm{HU}+\mathrm{AX}$ groups and was similar to that in the Con group (Fig. 2a).

\section{Ubiquitinated proteins expression}

Ubiquitinated proteins were measured as a marker of the activation of the ubiquitin-proteasome pathway that plays a key role in protein degradation due to muscle disuse such as HU [11]. Ubiquitinated proteins expression level in the HU group was significantly higher than that in the Con group; moreover, this level in the HU + AX group was significantly higher than that in the Con group but was significantly lower than that in the HU group. Ubiquitinated proteins expression level in the $\mathrm{HU}+\mathrm{ES}$ and $\mathrm{HU}+\mathrm{AX}+\mathrm{ES}$ groups were significantly lower than that in the $\mathrm{HU}$ and $\mathrm{HU}+\mathrm{AX}$ groups (Fig. 2b).

\section{SDH activity}

The representative images of SDH staining in each group are shown in Fig. 3a-e. SDH activity in the $\mathrm{HU}+\mathrm{AX}$ and $\mathrm{HU}+\mathrm{AX}+\mathrm{ES}$ groups were significantly higher than those in the Con, $\mathrm{HU}$, and $\mathrm{HU}+\mathrm{ES}$ groups (Fig. 3f). Integrated SDH activity, reflecting the total volume of mitochondria [36], was significantly lower in the $\mathrm{HU}$ and $\mathrm{HU}+\mathrm{ES}$ groups than in the Con group. Integrated SDH activity in the $\mathrm{HU}+\mathrm{AX}$ and $\mathrm{HU}+\mathrm{AX}+\mathrm{ES}$ groups was significantly higher than that in the HU group, whereas integrated SDH activity in the $\mathrm{HU}+\mathrm{AX}+\mathrm{ES}$ group was significantly higher than that in the $\mathrm{HU}+\mathrm{ES}$ group (Fig. 3g). 

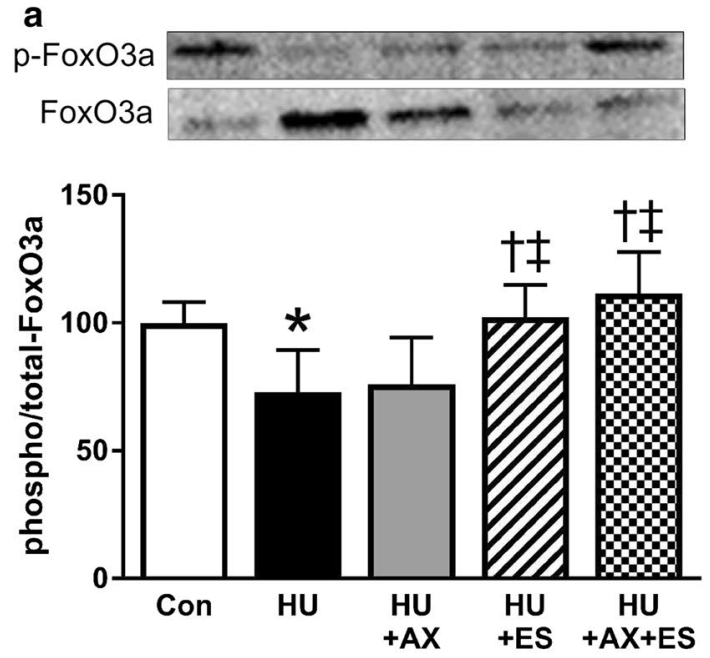

b
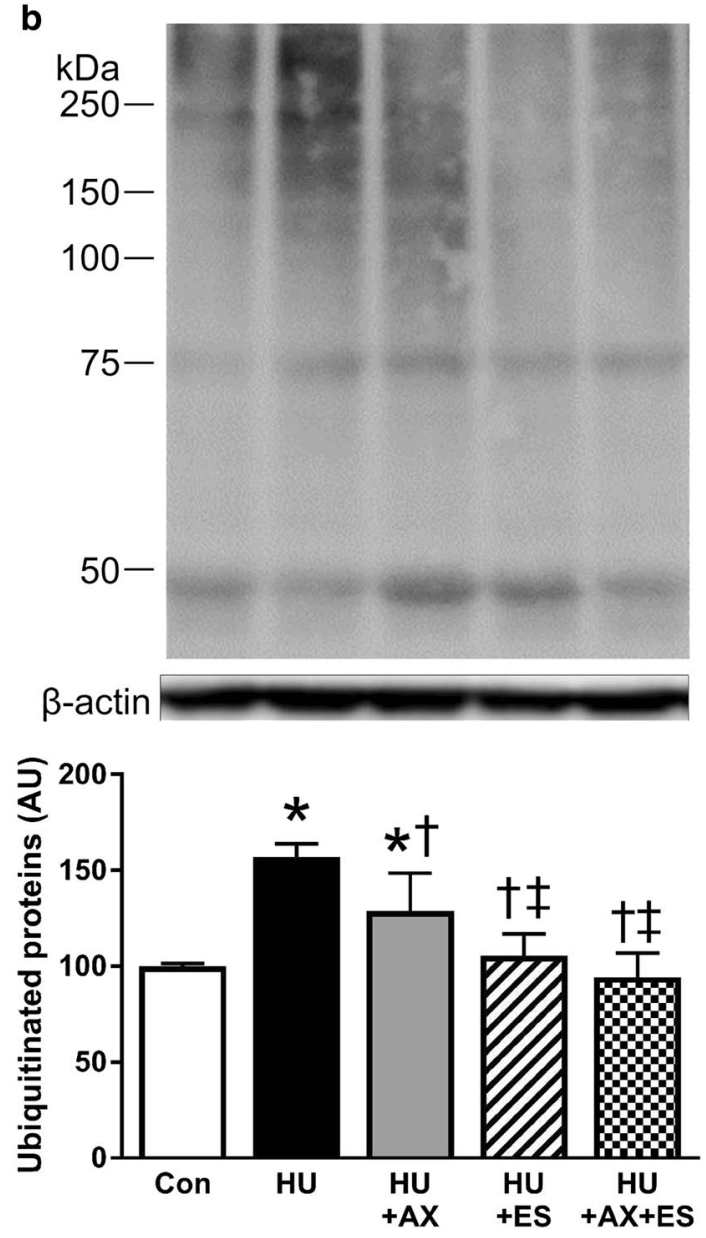

Fig. 2 Phosphorylated/total FoxO3a protein expression (a) and ubiquitinated proteins expression (b) in each group. Values indicate mean \pm SEM. The symbols $*,{ }^{\dagger},{ }^{\ddagger}$, and ${ }^{\S}$ significant differences from the Con, $\mathrm{HU}, \mathrm{HU}+\mathrm{AX}$, and $\mathrm{HU}+\mathrm{ES}$ groups, respectively, at $p<0.05$. Con control group, $H U$ hindlimb unloading group, $H U+A X$ hindlimb unloading plus astaxanthin treatment group, $H U+E S$ hindlimb unloading plus electrical stimulation group, $H U+A X+E S$ hindlimb unloading plus astaxanthin treatment with electrical stimulation group, SEM standard error of mean

\section{Capillary-to-fiber ratio}

The representative images of AP staining in each group are shown in Fig. 4a-e. C/F ratio in the HU group was significantly lower than that in the Con group. This level was significantly higher in the AX group than that in the HU group. Furthermore, $\mathrm{C} / \mathrm{F}$ ratio in the $\mathrm{HU}+\mathrm{AX}+\mathrm{ES}$ group was significantly higher than that in the $\mathrm{HU}$ and $\mathrm{HU}+\mathrm{ES}$ groups (Fig. 4f).

\section{PGC-1a protein expression}

PGC- $1 \alpha$ protein expression level in the HU group was significantly lower than that in the Con group. This level was significantly higher in the AX group than that in the HU group. Furthermore, PGC-1 $\alpha$ protein expression in the $\mathrm{HU}+\mathrm{AX}+\mathrm{ES}$ group was significantly higher than that in the $\mathrm{HU}$ and $\mathrm{HU}+\mathrm{ES}$ groups (Fig. 5).

\section{ROS production and SOD-1 protein expression}

We measured DHE fluorescence intensity and SOD-1 protein expression as an indicator of oxidative stress as previously described [15, 18, 20, 21, 34]. ROS production was assessed by measuring the DHE fluorescence intensity in the nucleus. DHE fluorescence intensity was normalized as fold of the corresponding DAPI fluorescence intensity. From the representative images of muscle cross sections stained with DHE, DHE fluorescence intensities of the HU and $\mathrm{HU}+\mathrm{ES}$ groups appeared to be higher than those of the other groups (Fig. 6a). Indeed, DHE fluorescence intensities of the $\mathrm{HU}$ and $\mathrm{HU}+\mathrm{ES}$ groups were significantly higher than those of the Con, $\mathrm{HU}+\mathrm{AX}$, and $\mathrm{HU}+\mathrm{AX}+\mathrm{ES}$ groups. In addition, DHE fluorescence intensity in the $\mathrm{HU}+\mathrm{AX}$ and $\mathrm{HU}+\mathrm{AX}+\mathrm{ES}$ groups was similar to that in the Con group (Fig. 6b). The SOD-1 protein expression level in the HU group was significantly higher than that in the Con group. However, this level in the $\mathrm{HU}+\mathrm{AX}$ and $\mathrm{HU}+\mathrm{AX}+\mathrm{ES}$ groups was significantly lower than that in the $\mathrm{HU}$ and $\mathrm{HU}+\mathrm{ES}$ groups and was similar to that in the Con group. These results indicated a weaker effect of ES on oxidative stress caused by HU (Fig. 6c).

\section{Discussion}

The novel findings of the present study are as follows: (1) ES has a minimal impact on oxidative stress and decreased muscle oxidative capacity caused by HU; (2) the combination of AX supplementation and ES was effective for preventing both atrophy and decreased oxidative capacity within the unloaded soleus muscle. These results suggest that the combination therapy involving ES and AX is an 

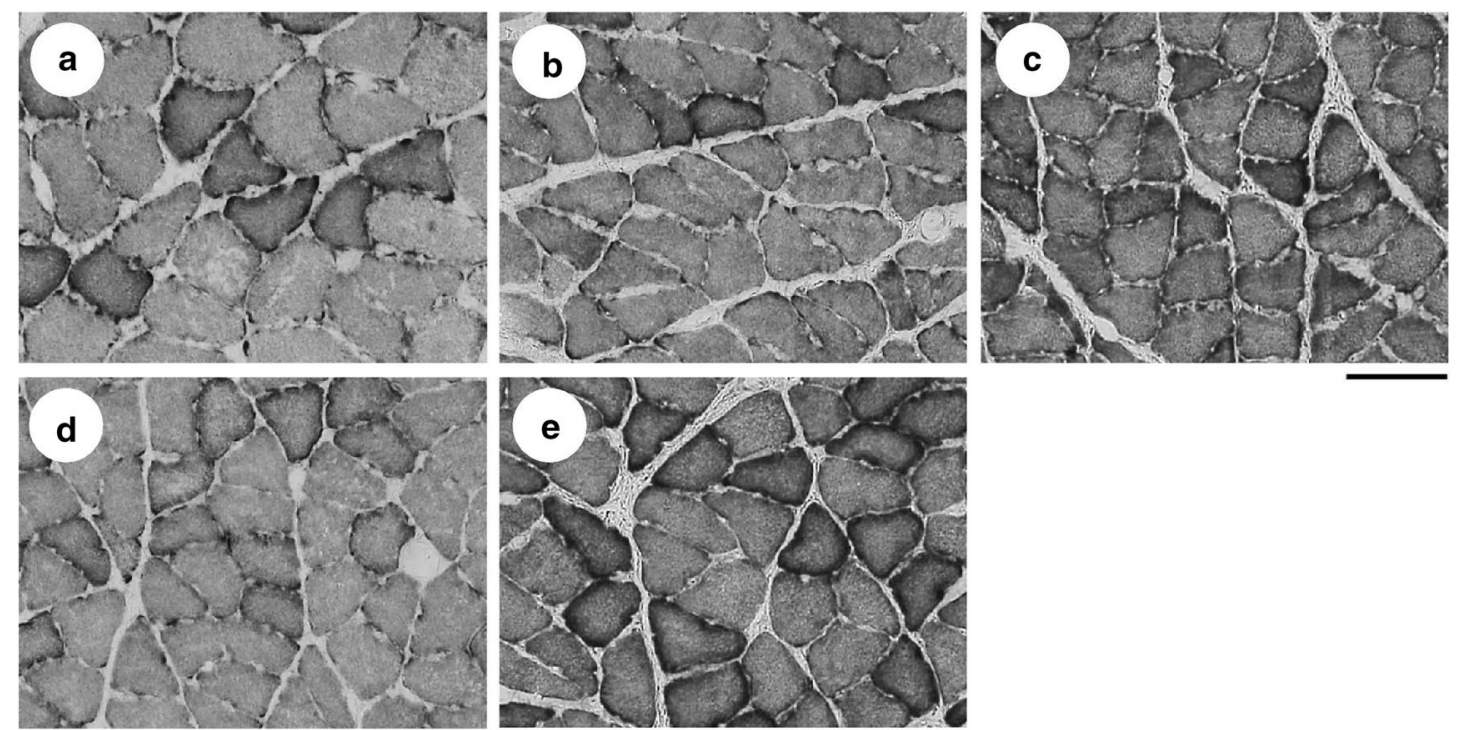

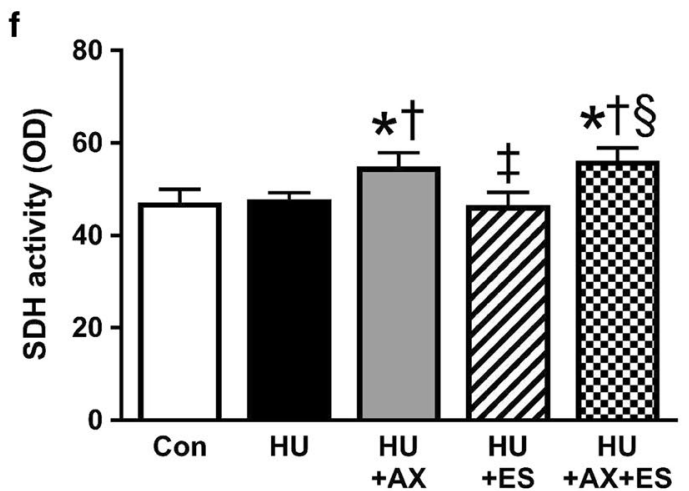

Fig. 3 Representative images of SDH staining of the soleus muscle in Con (a), $\mathrm{HU}(\mathbf{b}), \mathrm{HU}+\mathrm{AX}(\mathbf{c}), \mathrm{HU}+\mathrm{ES}(\mathbf{d})$, and $\mathrm{HU}+\mathrm{AX}+\mathrm{ES}$ (e) groups. SDH activity (f) and integrated SDH activity (g) in each group. Scale bar represents $100 \mu \mathrm{m}$. Values indicate mean \pm SEM. The symbols $*, \dagger, \ddagger$, and ${ }^{\S}$ significant differences from the Con, HU, $\mathrm{HU}+\mathrm{AX}$, and $\mathrm{HU}+\mathrm{ES}$ groups, respectively, at $p<0.05$. Con control

effective countermeasure for reversing both muscle atrophy and decreased oxidative capacity caused by muscle disuse.

In the present study, $\mathrm{HU}$ resulted in reduced muscle mass, FCSA, and phosphorylated FoxO3a protein expression and elevated ubiquitinated proteins expression within the unloaded soleus muscle. A chronic decrease in muscle activity induces muscle atrophy in oxidative slow-twitch muscles such as the soleus muscle $[8,15,20,34]$. HU, which is a well-established in vivo model for hypokinesia and hypodynamia, causes decreased muscle mass and FCSA within the unloaded soleus muscle. It has been reported that protein degradation during muscle disuse is associated with the activation of the ubiquitin-proteasome pathway [11], resulting in increased ubiquitinated proteins expression. In addition, the activation (decreased phosphorylation) of FoxO3a, a key regulator of atrogene transcription, is involved in the

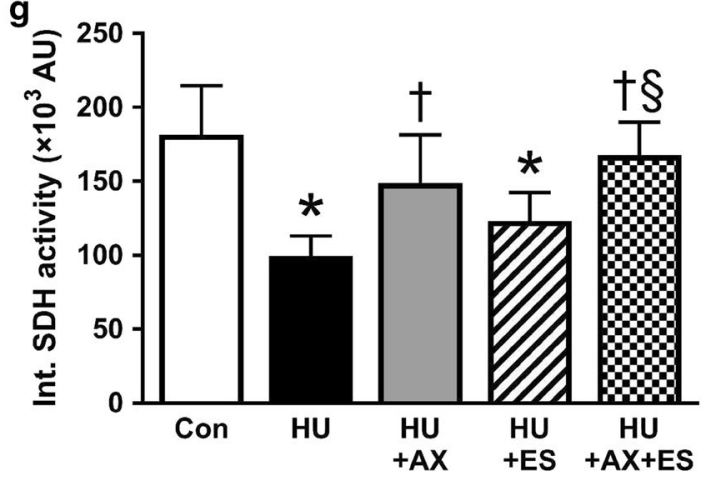

group, $H U$ hindlimb unloading group, $H U+A X$ hindlimb unloading plus astaxanthin treatment group, $H U+E S$ hindlimb unloading plus electrical stimulation group, $H U+A X+E S$ hindlimb unloading plus astaxanthin treatment with electrical stimulation group, SEM standard error of mean

ubiquitin-proteasome pathway [37]. Consistent with previous studies, our study demonstrated that HU induces muscle atrophy within the unloaded soleus muscle accompanied with the activation of the ubiquitin-proteasome pathway. Moreover, decreased oxidative capacity occurs in atrophied muscles and results in a decreased activity of mitochondrial rate-limiting enzymes involved in the TCA cycle [2]. Disuse-induced changes in muscle oxidative capacity result from the downregulation of PGC-1 $\alpha$ [2, 7], a key factor in the biogenesis and maintenance of mitochondria [38-40]. Decreased muscle oxidative capacity results in excessive ROS production, leading to oxidative stress [41]. Reportedly, oxidative stress damages mitochondria [42], suggesting that oxidative stress in disused muscle may result in further reduction of oxidative capacity. In addition, PGC- $1 \alpha$ plays a role in the mitochondrial antioxidant defense system of the 

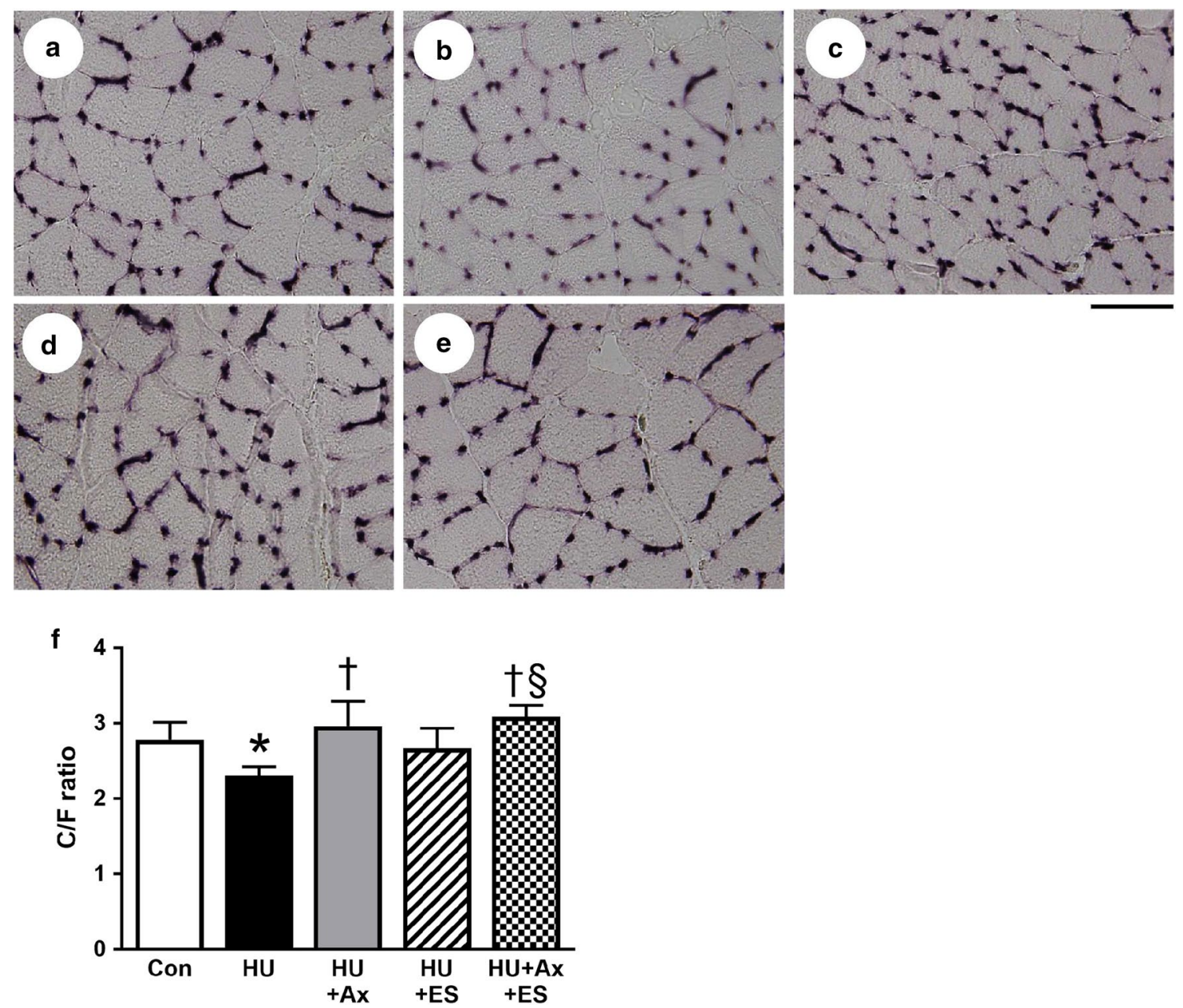

Fig. 4 Representative images of AP staining of the soleus muscle in Con (a), HU (b), HU + AX (c), HU + ES (d), and $\mathrm{HU}+\mathrm{AX}+\mathrm{ES}$ (e) groups. C/F ratio (f) in each group. Scale bar represents $100 \mu \mathrm{m}$. Values indicate mean \pm SEM. The symbols $*,{ }^{\dagger},{ }^{\ddagger}$, and ${ }^{\S}$ significant differences from the Con, $\mathrm{HU}, \mathrm{HU}+\mathrm{AX}$, and $\mathrm{HU}+\mathrm{ES}$ groups,

vascular endothelial cells; therefore, the downregulation of PGC- $1 \alpha$ under oxidative stress conditions results in further decline in mitochondrial function [43]. In the present study, $\mathrm{HU}$ reduced the activity of $\mathrm{SDH}$, a mitochondrial rate-limiting enzyme in the TCA cycle, and decreased PGC- $1 \alpha$ protein expression; however, it also increased ROS production as observed in DHE staining and increased SOD-1 protein expression within the unloaded soleus muscle. Our findings suggest an association between HU-induced decreased oxidative capacity and PGC- $1 \alpha$ downregulation, leading to oxidative stress in the unloaded soleus muscle.

AX exhibits a powerful antioxidant effect and prevents oxidative stress caused by disuse in the soleus muscle of rats [20,21]. In addition, our previous study revealed that AX suppresses disuse-associated reduction in the activity of mitochondrial rate-limiting enzymes such as SDH by restoring PGC-1 $\alpha$ protein expression [15]. Consistent with the respectively, at $p<0.05$. Con control group, $H U$ hindlimb unloading group, $H U+A X$ hindlimb unloading plus astaxanthin treatment group, $H U+E S$ hindlimb unloading plus electrical stimulation group, $H U+A X+E S$ hindlimb unloading plus astaxanthin treatment with electrical stimulation group, SEM standard error of mean

aforementioned previous findings, the present study demonstrated that AX treatment alone during $\mathrm{HU}$ had a strong positive effect on SDH activity and PGC- $1 \alpha$ protein expression, which appear to enhance oxidative capacity within the soleus muscle. Furthermore, as support for this observation, AX treatment during HU maintained muscle capillarization, which has a close relationship with muscle oxidative capacity [44], as expressed in $\mathrm{C} / \mathrm{F}$ ratio in the AX-treated soleus muscle, and these observations are correspond to our previous reports $[15,20]$. In contrast, $\mathrm{AX}$ treatment alone minimally affected muscle atrophy in the soleus muscle mass and FCSA in the present study; this finding is also consistent with that of previous studies [15, 20, 21]. However, ubiquitinated proteins expression was significantly lower in rats in the $\mathrm{HU}+\mathrm{AX}$ groups than that in those in the $\mathrm{HU}$ group. In addition, FoxO3a phosphorylation in the $\mathrm{HU}+\mathrm{AX}$ group was maintained at the same level as that in 

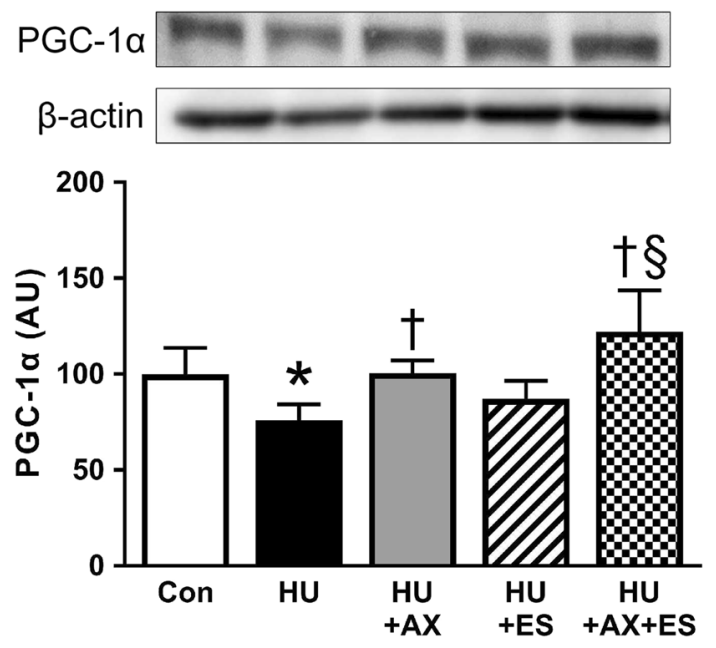

Fig. 5 PGC- $1 \alpha$ protein expression level in each group. Values indicate mean \pm SEM. The symbols $*, \dagger,{ }^{\ddagger}$, and ${ }^{\S}$ significant differences from the Con, HU, HU $+\mathrm{AX}$, and $\mathrm{HU}+\mathrm{ES}$ groups, respectively, at $p<0.05$. Con control group, $H U$ hindlimb unloading group, $H U+A X$ hindlimb unloading plus astaxanthin treatment group, $H U+E S$ hindlimb unloading plus electrical stimulation group, $H U+A X+E S$ hindlimb unloading plus astaxanthin treatment with electrical stimulation group, SEM standard error of mean

the Con group. Reportedly, PGC-1 $\alpha$ inhibits FoxO3a signaling, thus suppressing atrophy-specific gene transcription [45]. Thus, the observed reduction of ubiquitinated proteins expression may be associated with the suppressive effect of PGC- $1 \alpha$ on FoxO3a signaling. Furthermore, decreased muscle oxidative capacity results in AMPK activation [46], which upregulates proteolysis via the activation of FoxO3a and resultant ubiquitin-proteasome pathway [41, 46, 47]. In contrast, a previous study using PGC- $1 \alpha$ transgenic mice demonstrates that enhancing oxidative capacity in skeletal muscle during $\mathrm{HU}$ prevents the increased atrogene expression and thus attenuates muscle atrophy [7]. These results suggest that mitochondrial oxidative capacity modulates proteolysis during HU. Therefore, our findings suggest that AX treatment attenuates the activation of ubiquitin proteasome pathway during $\mathrm{HU}$ via its positive effect on mitochondrial oxidative capacity.

ES attenuates muscle atrophy caused by HU [22-26]. The underlying mechanism is partially associated with the suppression of the ubiquitin-proteasome pathway through mechanical stress evoked by ES. It was previously reported that ES during $\mathrm{HU}$ results in increased FoxO3a phosphorylation via mechanical stress-induced activation of Akt signaling, and thus decreased ubiquitinated proteins expression within the soleus muscles of HU rats [24]. Consistent with this finding, our results demonstrated the attenuating effect of ES on ubiquitin-proteasome pathway. Conversely, our findings showed that ES failed to attenuate HU-induced increase in DHE intensity, reflecting little impact of ES on HU-induced oxidative stress. Furthermore, ES did not restore integrated SDH activity, an indicator for mitochondrial total volume, reflecting the minimal effect of ES on decreased oxidative capacity. Increased ROS production has been reported to reduce muscle oxidative capacity [42], which may be associated with our findings. In all, our findings suggest that ES attenuates muscle atrophy by decreasing protein ubiquitination but does not suppress oxidative stress or HU-induced decline in muscle oxidative capacity. Although ES fully suppressed upregulation of ubiquitin-proteasome pathway, ES treatment had a minimal effect on FCSA in the HU + ES group in the present study. In the previous study, it was demonstrated that ES treatment could attenuate HU-induced decrease in FCSA via modulating ubiquitin-proteasome pathway [22-26]. The difference in the effect of ES on FCSA may be related to the different duration of $\mathrm{HU}$ exposure, i.e. 2 weeks of $\mathrm{HU}$ in the previous studies [22-26] versus a week of HU in the present study. It has been reported that in addition to ubiquitin-proteasome pathway-related proteolysis, myonuclear apoptosis also associates with unloading-induced decline in FCSA. HU-induced protein degradation reaches its peak at about 2 weeks after initiating unloading [11, 48, 49]. Conversely, it has been reported that in the early stages of $\mathrm{HU}$, increased apoptosisrelated protein and gene expression $[50,51]$ and myonuclear apoptosis [52] were observed within the unloaded muscle. Numerous studies suggested that oxidative stress has close relationship with myonuclear apoptosis [10, 18, 41]. In the present study, ES treatment alone exerted no suppressive effect on oxidative stress within the ES-treated soleus muscle. Therefore, it was probable that ES treatment was insufficient to normalize myonuclear apoptosis in the early stages of $\mathrm{HU}$, which might associate with a minimal effect on FCSA in the HU + ES group despite the suppression of protein ubiquitination. Nonetheless, we did not investigate myonuclear apoptosis within the muscles in the present study, further studies will be required to clarify the specific effects of ES on myonucleus within unloaded muscle.

It has been reported that $\mathrm{HU}$ is associated with muscle atrophy [11] and decreased oxidative capacity [7] and the mechanisms underlying these changes are different. Consistent with these reports, we showed that antioxidant AX treatment had a positive effect on oxidative stress while it failed to attenuate increased protein ubiquitination due to $\mathrm{HU}$, while ES treatment exhibited the opposite trend. These findings suggest that intervention with AX or ES alone is insufficient for reversing the alterations caused by HU such as muscle atrophy, decreased oxidative capacity and capillary regression. However, the combination of AX and ES treatments significantly attenuated the HUinduced decline in FCSA. With the combinational treatment, both protein ubiquitination and oxidative stress were significantly suppressed within the unloaded soleus muscle 

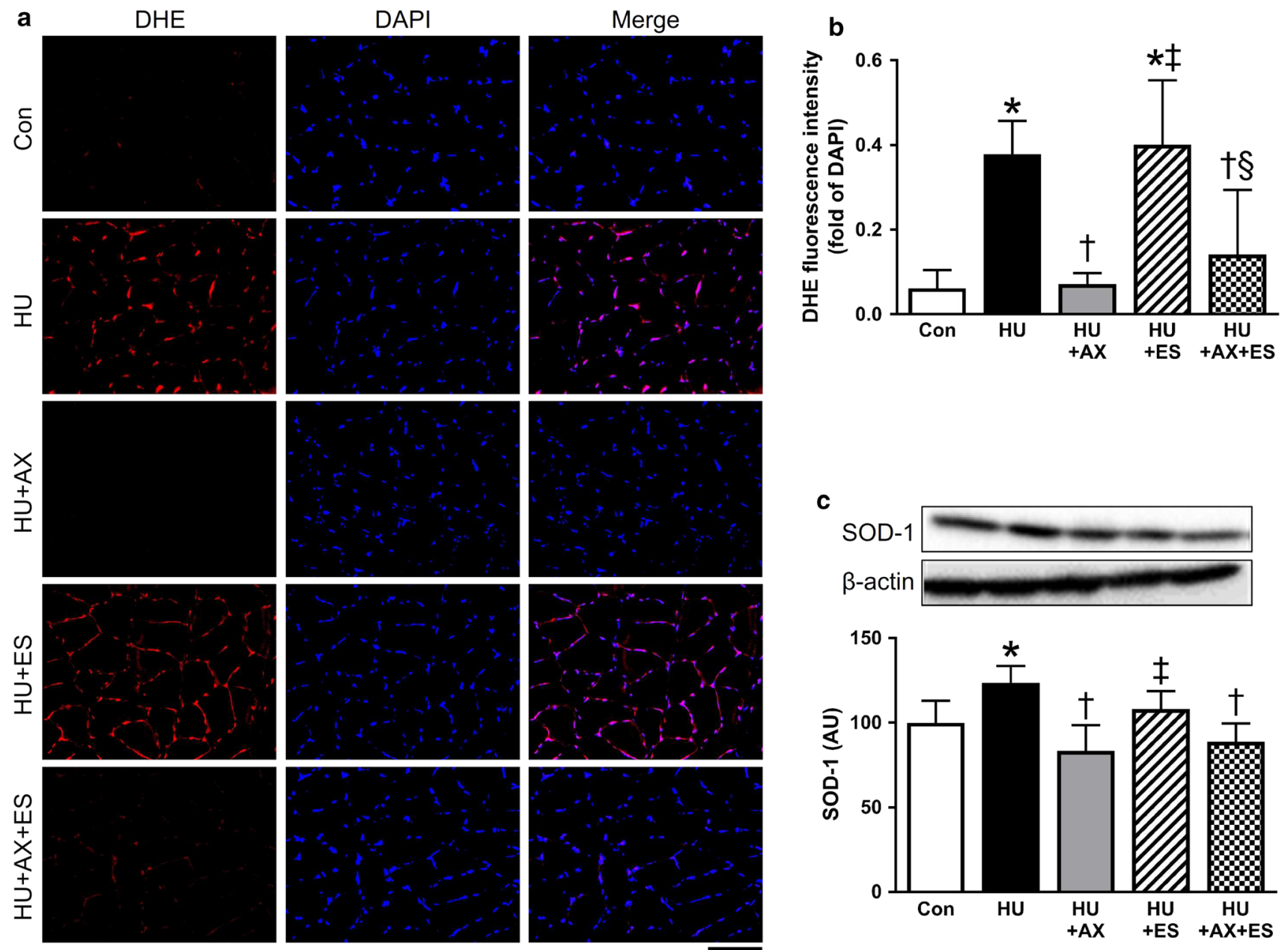

Fig. 6 Representative images of dihydroethidium (DHE; red) and DAPI (blue) staining and merged images of the soleus muscle in each group (a). The graph for DHE fluorescence intensity (b) depicts ratio of DHE and corresponding DAPI fluorescence intensity, which was measured with BZ-X Analyzer. Protein expression level of SOD-1 (c) in each group. Scale bar represents $100 \mu \mathrm{m}$. Values indicate mean \pm SEM. The symbols $*, \dagger,{ }^{\ddagger}$, and ${ }^{\S}$ significant differences

in the present study. It has been reported that oxidative stress decreases protein synthesis signaling [9]. Moreover, oxidative stress associates with apoptotic signaling as well. Yoshihara et al. [18] reported that antioxidative AX treatment ameliorated HU-related myonuclear apoptosis and resultant muscle atrophy. Therefore, there might be possibility that AX treatment contributed to optimization of protein synthesis signaling activated by ES and attenuation of myonuclear apoptosis due to HU by suppressing oxidative stress in the unloaded muscle, which helped to reduce atrophy in the soleus muscle treated with the combinational interventions. Although there was almost completely prevention of increased oxidative stress and ubiquitin proteasome pathway, full recovery of FCSA was not obtained by the cotreatment. Because multiple factors from the Con, $\mathrm{HU}, \mathrm{HU}+\mathrm{AX}$, and $\mathrm{HU}+\mathrm{ES}$ groups, respectively, at $p<0.05$. Con control group, $H U$ hindlimb unloading group, $H U+A X$ hindlimb unloading plus astaxanthin treatment group, $H U+E S$ hindlimb unloading plus electrical stimulation group, $H U+A X+E S$ hindlimb unloading plus astaxanthin treatment with electrical stimulation group, SEM standard error of mean (colour figure online)

are involved in muscle atrophy $[53,54]$, in the future study further investigation will be required to clarify the effects of the combination of $\mathrm{AX}$ and $\mathrm{ES}$ treatment on each factor causing atrophy. Consequently, we suggest that combination of AX supplementation and ES could attenuate both muscle atrophy and decreased oxidative enzymatic activity due to $\mathrm{HU}$ and restore key regulators such as FoxO3a phosphorylation, oxidative stress, and PGC- $1 \alpha$ to near or above control levels. These results strongly suggest that the combination treatment involving AX and ES is effective for preventing unloading-induced decreased oxidative capacity and muscle atrophy, respectively, via different pathways. Furthermore, these findings suggest that the combination treatment is an effective therapeutic strategy for preventing various muscle dysfunctions associated 
with protein degradation and decreased oxidative capacity including cancer cachexia, diabetes, and denervation.

In conclusion, the current study demonstrates that the combination treatment involving ES and AX is effective for attenuating both muscle atrophy and oxidative capacity via different mechanisms. The findings of this study also suggest a potential use for this combination treatment along with mechanical loading and nutritional support for preventing various muscle dysfunctions caused by muscle disuse.

Acknowledgements This study was supported by Grants-in-Aid for Scientific Research (15K16516 and 17K18028) from the Japanese Ministry of Education, Culture, Sports, Science and Technology.

Author contribution MK and HF conceived and designed the experiments. MK, MT, and RN performed the experiments. MK, MT and $\mathrm{RN}$ analyzed the data. MK, MT, NM, and HF contributed by providing regents, materials and analysis tools. MK, MT, NM, and HF interpreted the data and wrote the paper. All authors approved the final version of the manuscript.

\section{Compliance with ethical standards}

Conflict of interest The authors declare that they have no conflicts of interest.

Ethical approval All applicable international, national, and/or institutional guidelines for the care and use of animals were followed. All procedures performed in studies involving animals were in accordance with the ethical standards of the institution or practice at which the studies were conducted. This article does not contain any studies with human participants performed by any of the authors.

\section{References}

1. Morey ER, Sabelman EE, Turner RT, Baylink DJ (1979) A new rat model simulating some aspects of space flight. Physiologist 22:S23-S24

2. Nagatomo F, Fujino H, Kondo H, Suzuki H, Kouzaki M, Takeda I, Ishihara A (2011) PGC-1alpha and FOXO1 mRNA levels and fiber characteristics of the soleus and plantaris muscles in rats after hindlimb unloading. Histol Histopathol 26:1545-1553

3. Wang J, Wang F, Zhang P, Liu H, He J, Zhang C, Fan M, Chen X (2017) PGC-1alpha over-expression suppresses the skeletal muscle atrophy and myofiber-type composition during hindlimb unloading. Biosci Biotechnol Biochem 81:500-513

4. Fujino H, Kohzuki H, Takeda I, Kiyooka T, Miyasaka T, Mohri S, Shimizu J, Kajiya F (2005) Regression of capillary network in atrophied soleus muscle induced by hindlimb unweighting. J Appl Physiol (1985) 98:1407-1413

5. Hirayama Y, Nakanishi R, Maeshige N, Fujino H (2017) Preventive effects of nucleoprotein supplementation combined with intermittent loading on capillary regression induced by hindlimb unloading in rat soleus muscle. Physiol Rep 5(4):e13134

6. Roudier E, Gineste C, Wazna A, Dehghan K, Desplanches D, Birot O (2010) Angio-adaptation in unloaded skeletal muscle: new insights into an early and muscle type-specific dynamic process. J Physiol 588:4579-4591

7. Cannavino J, Brocca L, Sandri M, Bottinelli R, Pellegrino MA (2014) PGC1-alpha over-expression prevents metabolic alterations and soleus muscle atrophy in hindlimb unloaded mice. J Physiol 592:4575-4589

8. Fujino H, Ishihara A, Murakami S, Yasuhara T, Kondo H, Mohri S, Takeda I, Roy RR (2009) Protective effects of exercise preconditioning on hindlimb unloading-induced atrophy of rat soleus muscle. Acta Physiol (Oxf) 197:65-74

9. Gao Y, Arfat Y, Wang H, Goswami N (2018) Muscle atrophy induced by mechanical unloading: mechanisms and potential countermeasures. Front Physiol 9:235

10. Powers SK, Kavazis AN, McClung JM (2007) Oxidative stress and disuse muscle atrophy. J Appl Physiol (1985) 102:2389-2397

11. Jackman RW, Kandarian SC (2004) The molecular basis of skeletal muscle atrophy. Am J Physiol Cell Physiol 287:C834-C843

12. Andrianjafiniony T, Dupre-Aucouturier S, Letexier D, Couchoux H, Desplanches D (2010) Oxidative stress, apoptosis, and proteolysis in skeletal muscle repair after unloading. Am J Physiol Cell Physiol 299:C307-C315

13. Wagatsuma A, Kotake N, Kawachi T, Shiozuka M, Yamada S, Matsuda R (2011) Mitochondrial adaptations in skeletal muscle to hindlimb unloading. Mol Cell Biochem 350:1-11

14. Desaphy JF, Pierno S, Liantonio A, Giannuzzi V, Digennaro C, Dinardo MM, Camerino GM, Ricciuti P, Brocca L, Pellegrino MA, Bottinelli R, Camerino DC (2010) Antioxidant treatment of hindlimb-unloaded mouse counteracts fiber type transition but not atrophy of disused muscles. Pharmacol Res 61:553-563

15. Kanazashi M, Tanaka M, Murakami S, Kondo H, Nagatomo F, Ishihara A, Roy RR, Fujino H (2014) Amelioration of capillary regression and atrophy of the soleus muscle in hindlimb-unloaded rats by astaxanthin supplementation and intermittent loading. Exp Physiol 99:1065-1077

16. Jaffer OA, Carter AB, Sanders PN, Dibbern ME, Winters CJ, Murthy S, Ryan AJ, Rokita AG, Prasad AM, Zabner J, Kline JN, Grumbach IM, Anderson ME (2015) Mitochondrial-targeted antioxidant therapy decreases transforming growth factor-betamediated collagen production in a murine asthma model. Am J Respir Cell Mol Biol 52:106-115

17. Min K, Smuder AJ, Kwon OS, Kavazis AN, Szeto HH, Powers SK (2011) Mitochondrial-targeted antioxidants protect skeletal muscle against immobilization-induced muscle atrophy. J Appl Physiol (1985) 111:1459-1466

18. Yoshihara T, Yamamoto Y, Shibaguchi T, Miyaji N, Kakigi R, Naito H, Goto K, Ohmori D, Yoshioka T, Sugiura T (2017) Dietary astaxanthin supplementation attenuates disuse-induced muscle atrophy and myonuclear apoptosis in the rat soleus muscle. J Physiol Sci 67:181-190

19. Brocca L, Pellegrino MA, Desaphy JF, Pierno S, Camerino DC, Bottinelli R (2010) Is oxidative stress a cause or consequence of disuse muscle atrophy in mice? A proteomic approach in hindlimb-unloaded mice. Exp Physiol 95:331-350

20. Kanazashi M, Okumura Y, Al-Nassan S, Murakami S, Kondo H, Nagatomo F, Fujita N, Ishihara A, Roy RR, Fujino H (2013) Protective effects of astaxanthin on capillary regression in atrophied soleus muscle of rats. Acta Physiol (Oxf) 207:405-415

21. Maezawa T, Tanaka M, Kanazashi M, Maeshige N, Kondo H, Ishihara A, Fujino H (2017) Astaxanthin supplementation attenuates immobilization-induced skeletal muscle fibrosis via suppression of oxidative stress. J Physiol Sci 67:603-611

22. Tanaka M, Hirayama Y, Fujita N, Fujino H (2013) Comparison of premodulated interferential and pulsed current electrical stimulation in prevention of deep muscle atrophy in rats. J Mol Histol 44:203-211

23. Tanaka M, Hirayama Y, Fujita N, Fujino H (2014) Electrical stimulation using sine waveform prevents unloading-induced muscle atrophy in the deep calf muscles of rat. Acta Histochem 116:1192-1198 
24. Tanaka M, Nakanishi R, Murakami S, Fujita N, Kondo H, Ishihara A, Roy RR, Fujino H (2016) Effectiveness of daily eccentric contractions induced via kilohertz frequency transcutaneous electrical stimulation on muscle atrophy. Acta Histochem 118:56-62

25. Fujita N, Murakami S, Arakawa T, Miki A, Fujino H (2011) The combined effect of electrical stimulation and resistance isometric contraction on muscle atrophy in rat tibialis anterior muscle. Bosn J Basic Med Sci 11:74-79

26. Fujita N, Murakami S, Fujino H (2011) The combined effect of electrical stimulation and high-load isometric contraction on protein degradation pathways in muscle atrophy induced by hindlimb unloading. J Biomed Biotechnol 2011:401493

27. Booth FW, Ruegsegger GN, Toedebusch RG, Yan Z (2015) Endurance exercise and the regulation of skeletal muscle metabolism. Prog Mol Biol Transl Sci 135:129-151

28. Hood DA (1985) (2001) Invited Review: contractile activityinduced mitochondrial biogenesis in skeletal muscle. J Appl Physiol (1985) 90:1137-1157

29. Perry CG, Lally J, Holloway GP, Heigenhauser GJ, Bonen A, Spriet LL (2010) Repeated transient mRNA bursts precede increases in transcriptional and mitochondrial proteins during training in human skeletal muscle. J Physiol 588:4795-4810

30. Morey-Holton ER, Globus RK (2002) Hindlimb unloading rodent model: technical aspects. J Appl Physiol (1985) 92:1367-1377

31. Kondo H, Fujino H, Murakami S, Tanaka M, Kanazashi M, Nagatomo F, Ishihara A, Roy RR (2015) Low-intensity running exercise enhances the capillary volume and pro-angiogenic factors in the soleus muscle of type 2 diabetic rats. Muscle Nerve 51:391-399

32. Bouitbir J, Charles AL, Rasseneur L, Dufour S, Piquard F, Geny B, Zoll J (2011) Atorvastatin treatment reduces exercise capacities in rats: involvement of mitochondrial impairments and oxidative stress. J Appl Physiol (1985) 111:1477-1483

33. Cozzoli A, Nico B, Sblendorio VT, Capogrosso RF, Dinardo MM, Longo V, Gagliardi S, Montagnani M, De Luca A (2011) Enalapril treatment discloses an early role of angiotensin II in inflammation- and oxidative stress-related muscle damage in dystrophic mdx mice. Pharmacol Res 64:482-492

34. Tanaka M, Kanazashi M, Maeshige N, Kondo H, Ishihara A, Fujino H (2018) Protective effects of Brazilian propolis supplementation on capillary regression in the soleus muscle of hindlimb-unloaded rats. J Physiol Sci 69:223-233

35. Li Z, Wang Y, Man RY, Vanhoutte PM (2013) Upregulation of heme oxygenase-1 potentiates EDH-type relaxations in the mesenteric artery of the spontaneously hypertensive rat. Am J Physiol Heart Circ Physiol 305:H1471-H1483

36. van der Laarse WJ, Diegenbach PC, Elzinga G (1989) Maximum rate of oxygen consumption and quantitative histochemistry of succinate dehydrogenase in single muscle fibres of Xenopus laevis. J Muscle Res Cell Motil 10:221-228

37. Sandri M, Sandri C, Gilbert A, Skurk C, Calabria E, Picard A, Walsh K, Schiaffino S, Lecker SH, Goldberg AL (2004) Foxo transcription factors induce the atrophy-related ubiquitin ligase atrogin-1 and cause skeletal muscle atrophy. Cell 117:399-412

38. Geng T, Li P, Okutsu M, Yin X, Kwek J, Zhang M, Yan Z (2010) PGC-1alpha plays a functional role in exercise-induced mitochondrial biogenesis and angiogenesis but not fiber-type transformation in mouse skeletal muscle. Am J Physiol Cell Physiol 298:C572-C579

39. Olesen J, Kiilerich K, Pilegaard H (2010) PGC-1alpha-mediated adaptations in skeletal muscle. Pflugers Arch 460:153-162
40. Takemura A, Roy RR, Yoshihara I, Ishihara A (2017) Unloadinginduced atrophy and decreased oxidative capacity of the soleus muscle in rats are reversed by pre- and postconditioning with mild hyperbaric oxygen. Physiol Rep 5(14):e13353

41. Powers SK, Wiggs MP, Duarte JA, Zergeroglu AM, Demirel HA (2012) Mitochondrial signaling contributes to disuse muscle atrophy. Am J Physiol Endocrinol Metab 303:E31-E39

42. Bonnard C, Durand A, Peyrol S, Chanseaume E, Chauvin MA, Morio B, Vidal H, Rieusset J (2008) Mitochondrial dysfunction results from oxidative stress in the skeletal muscle of diet-induced insulin-resistant mice. J Clin Investig 118:789-800

43. Valle I, Alvarez-Barrientos A, Arza E, Lamas S, Monsalve M (2005) PGC-1alpha regulates the mitochondrial antioxidant defense system in vascular endothelial cells. Cardiovasc Res 66:562-573

44. Maxwell LC, White TP, Faulkner JA (1980) Oxidative capacity, blood flow, and capillarity of skeletal muscles. J Appl Physiol Respir Environ Exerc Physiol 49:627-633

45. Sandri M, Lin J, Handschin C, Yang W, Arany ZP, Lecker SH, Goldberg AL, Spiegelman BM (2006) PGC-1alpha protects skeletal muscle from atrophy by suppressing FoxO3 action and atrophy-specific gene transcription. Proc Natl Acad Sci USA 103:16260-16265

46. Liu J, Peng Y, Wang X, Fan Y, Qin C, Shi L, Tang Y, Cao K, Li H, Long J, Liu J (2016) Mitochondrial dysfunction launches dexamethasone-induced skeletal muscle atrophy via AMPK/FOXO3 signaling. Mol Pharm 13:73-84

47. Romanello V, Sandri M (2010) Mitochondrial biogenesis and fragmentation as regulators of muscle protein degradation. Curr Hypertens Rep 12:433-439

48. Edgerton VR, Roy RR (1994) Neuromuscular adaptation to actual and simulated weightlessness. Adv Space Biol Med 4:33-67

49. Thomason DB, Booth FW (1990) Atrophy of the soleus muscle by hindlimb unweighting. J Appl Physiol (1985) 68:1-12

50. Dupont-Versteegden EE, Strotman BA, Gurley CM, Gaddy D, Knox M, Fluckey JD, Peterson CA (2006) Nuclear translocation of EndoG at the initiation of disuse muscle atrophy and apoptosis is specific to myonuclei. Am J Physiol Regul Integr Comp Physiol 291:R1730-R1740

51. Ferreira R, Neuparth MJ, Vitorino R, Appell HJ, Amado F, Duarte JA (2008) Evidences of apoptosis during the early phases of soleus muscle atrophy in hindlimb suspended mice. Physiol Res 57:601-611

52. Leeuwenburgh C, Gurley CM, Strotman BA, Dupont-Versteegden EE (2005) Age-related differences in apoptosis with disuse atrophy in soleus muscle. Am J Physiol Regul Integr Comp Physiol 288:R1288-R1296

53. Bonaldo P, Sandri M (2013) Cellular and molecular mechanisms of muscle atrophy. Dis Model Mech 6:25-39

54. Scicchitano BM, Dobrowolny G, Sica G, Musaro A (2018) Molecular insights into muscle homeostasis, atrophy and wasting. Curr Genom 19:356-369

Publisher's Note Springer Nature remains neutral with regard to jurisdictional claims in published maps and institutional affiliations. 